\title{
"Tô sentindo nada": percepçôes de pacientes idosos sobre o tratamento da hipertensão arterial sistêmica
}

\section{$1{ }^{1}$ Leonardo Oliveira Leão e Silva, ${ }^{2}$ Marina Mendes Soares, ${ }^{3}$ Mericley Angela de Oliveira, ${ }^{4}$ Suely Maria Rodrigues, ${ }^{5}$ Carla Jorge Machado, ${ }^{6}$ Carlos Alberto Dias I}

Resumo: Este artigo objetivou identificar as percepções de pacientes idosos hipertensos em torno da terapêutica, relacionadas à adesão ou não ao tratamento medicamentoso. A amostra qualitativa foi constituída de 16 idosos, de ambos os sexos, com 60 anos ou mais, residentes em dois distritos rurais do município de Governador Valadares, que utilizavam algum medicamento para controle da pressão arterial e que tinham estado funcional independente e função cognitiva preservada. O método de coleta de dados utilizado foi a entrevista aberta em profundidade, com auxílio de um gravador de voz digital, realizada entre os meses de junho e setembro de 2010. A apuração das informações obtidas foi realizada segundo a técnica da análise de conteúdo, de Bardin. Contemplaram-se as categorias: Prática e conhecimento, Medidas Higienodietéticas (Alimentação, Exercícios físicos, Estresse e nervosismo) e Tratamento medicamentoso. Os resultados mostraram que os idosos possuem conhecimentos adequados sobre hipertensão e tratamento, mas são impulsionados, durante a terapêutica, a agirem segundo suas opiniōes e experiências socialmente compartilhadas. Ademais, os idosos hipertensos nãoaderentes ao tratamento tendem a considerar a hipertensão como uma doença aguda, sintomática e ligada a estados emocionais. Conclui-se que, devido a essa percepção equivocada, torna-se necessário reavaliar as orientaçōes fornecidas aos hipertensos pela equipe de saúde, devendose criar alternativas viáveis de promoção da saúde, para que ocorram mudanças de atitudes e práticas quanto à doença.

\author{
1 Enfermeiro. Doutorando \\ em Saúde Coletiva pela \\ Universidade Federal do Rio de \\ Janeiro. Endereço eletrônico: \\ leooliveiraleao@hotmail.com. \\ 2 Psicóloga. Mestranda em \\ Gestão Integrada do território \\ pela Universidade Vale do Rio \\ Doce. Endereço eletrônico: \\ marinamantena@hotmail.com \\ ${ }^{3}$ Enfermeira. Especialista em \\ Saúde Coletiva. Endereço \\ eletrônico: mericleyoliveira@ \\ hotmail.com \\ ${ }^{4}$ Cirurgiã-dentista. Doutora em \\ Saúde Coletiva. Professora da \\ Universidade Vale do Rio Doce. \\ Endereço eletrônico: badi@ \\ univale.br \\ ${ }^{5}$ Economista. Doutora em \\ Dinâmica Populacional. \\ Professora da Universidade \\ Federal de Minas Gerais. \\ Endereço eletrônico: \\ cjmachado@terra.com.br \\ ${ }^{6}$ Psicólogo. Doutor em \\ Psicologia Clinica. Professor da \\ Universidade Vale do Rio Doce. \\ Endereço eletrônico: cdias@ \\ univale.br
}

Recebido em: 18/06/2012. Aprovado em: 21/02/2013. 


\section{Introdução}

Segundo a Organização Mundial da Saúde, estima-se que 6,4\% da população mundial seja composta por pessoas idosas, ou seja, 65 anos ou mais, nos países desenvolvidos, e 60 anos ou mais nos países em desenvolvimento (WHO, 2008). Este é o segmento da população que mais cresce em países desenvolvidos, já que a cada mês o número de pessoas que se tornam idosas chega a 800 mil (WHO, 2008). O Brasil, à semelhança, está passando por um processo de envelhecimento populacional. Estudos projetaram um aumento da população brasileira acima de 60 anos na ordem de 15 vezes, entre os anos de 1950 e 2025 (CARVALHO; GARCIA, 2003). Para o mesmo período, acredita-se que o crescimento dessa população no mundo será de cinco vezes, e esse aumento colocará o Brasil no ano 2025 como a sexta população de idosos do mundo (IBGE, 2003).

$\mathrm{O}$ envelhecimento da população implica uma mudança no perfil epidemiológico. Uma população jovem, com maior incidência de doenças infecciosas, se torna uma população na qual predominam as doenças crônicas, características de uma população envelhecida (CARVALHO et al., 2008). Dentre as doenças crônicas que atingem os idosos, a hipertensão arterial sistêmica (HAS) possui grande incidência e prevalência e baixas taxas de controle (CHOBANIAN et al., 2003) Estudos indicam que a prevalência da HAS entre os indivíduos com mais de 60 anos é alta, variando entre 51 a 61\% dessa população (FIRMO et al., 2003; ZAITUNE et al., 2006).

A HAS, considerada uma "assassina silenciosa" (SANTOS et al., 2005), é o maior problema médico-social dos países desenvolvidos e em muitos dos emergentes. Mesmo conhecendo-se a eficácia, a efetividade e a eficiência de várias das medidas preventivas e de controle disponíveis, sejam ou não farmacológicas, a HAS continuará, por décadas, representando um dos maiores desafios em saúde e um dos maiores ônus para o próprio hipertenso e para a sociedade (SANTOS et al., 2005).

O tratamento adequado é um passo fundamental para o controle da HAS e suas complicações. Este pode consistir tanto na adoção de estilo de vida saudável, quanto no uso de medicação ou a associação de ambos, com base nas evidências científicas e consensos de especialistas. No entanto, apesar da grande variedade e disponibilidade dos agentes anti-hipertensivos disponíveis para o tratamento da HAS, menos de $1 / 3$ dos pacientes hipertensos adultos têm sua pressão 
adequadamente controlada. Dados epidemiológicos da população dos Estados Unidos da America têm evidenciado que, de $54 \%$ das pessoas que sabem ser hipertensas e recebem tratamento para esta condição, apenas $27 \%$ têm a sua pressão arterial (PA) controlada em níveis recomendados (JNC, 1997).

Para que o controle e a adesão da HAS ocorram, torna-se importante que os profissionais de saúde sejam capazes de ir além do mero conhecimento dos fatores objetivos que influem nesse controle. Ou seja, os profissionais devem procurar conhecer, em profundidade, o modo como os sujeitos pertencentes a este grupo populacional percebem a doença e a terapêutica. Pesquisas indicam que este conhecimento pode criar condições para identificar fatores que impedem os idosos de aderir ao tratamento (PERES et al., 2003). Portanto, o presente estudo teve por objetivo identificar as percepções de pacientes idosos hipertensos em torno da terapêutica relacionadas à adesão ou não ao tratamento medicamentoso em um município de médio porte do Estado de Minas Gerais em 2010. ${ }^{1}$

\section{Metodologia}

A amostra inicial foi constituída de 99 idosos, com 60 anos ou mais, residentes em dois distritos rurais do município de Governador Valadares, Estado de Minas Gerais. Foram considerados para o estudo indivíduos de ambos os sexos, constantes nos arquivos do Hiperdia no mês de maio de 2010, que utilizavam algum medicamento para controle da PA e que tinham estado funcional independente e função cognitiva preservada para responder ao questionário utilizado nesta fase da pesquisa. Para a avaliação de tais condições, contou-se com registros dos prontuários arquivados nas Estratégias Saúde da Família (ESF) dos distritos de Chonin de Cima e Chonin de Baixo e com o parecer de profissionais de saúde - médicos e enfermeiros - que atuam nestas ESFs.

Com o objetivo de identificar questões relativas à vivência diária e compreender significados das práticas individuais relacionados ao uso dos medicamentos, foi aplicado o teste de Morisky-Green (1986). Este consiste de um questionário com quatro questóes que verificam se o paciente se esqueceu de administrar seu medicamento, se administrou na hora prescrita e se deixou de administrar dependendo de como se sentia. As respostas foram pontuadas em "sim" e "não". Segundo o teste, considera-se aderente ao tratamento proposto o paciente que 
obteve pontuação máxima de quatro pontos, e não aderente aquele que obteve três pontos ou menos (MORISKY et al., 1986).

Os resultados do teste de Morisky indicaram que 62,6\% dos idosos não aderem ao tratamento anti-hipertensivo prescrito pela equipe médica. Tomandose como referência esses resultados, os idosos foram divididos em dois grupos: G1, com 37 idosos aderentes ao tratamento, e G2, com 62 idosos não aderentes ao tratamento.

Após esta etapa, foram sorteados oito participantes de cada grupo, totalizando 16 idosos que fizeram parte da amostra final do estudo. A definição do número de indivíduos não se fez com base em amostragem probabilística, sendo o número de sujeitos delimitado pela saturação dos discursos (BAUER ; AARTS, 2002).

O método de coleta de dados utilizado foi a entrevista aberta em profundidade. A técnica de entrevista é indicada quando o pesquisador deseja obter o maior número possível de informaçoes sobre determinado tema, segundo a visão do entrevistado, e também para obter maior detalhamento do assunto em questão. Em relação à sua estruturação, o entrevistador introduz o tema e o entrevistado tem liberdade para discorrer sobre o tema sugerido. É uma forma de poder explorar mais amplamente uma questão. As perguntas são respondidas dentro de uma conversação informal (MINAYO, 1993).

A coleta de dados foi realizada entre os meses de junho e setembro de 2010. Foi realizada individualmente nas residências dos participantes, em ambiente reservado, que apresentou boa iluminação, ventilação adequada, cadeiras confortáveis, tranquilidade e silêncio, procurando assegurar a privacidade dos participantes. Durante a realização da entrevista, o entrevistador fazia a pergunta prevista e assumia uma postura de instigador, explorando ao máximo a fala do informante. Em todas as entrevistas manteve-se um caráter informal, a fim de que o idoso se sentisse à vontade para relatar suas opinióes.

No intuito de apresentar fidelidade quanto ao registro das respostas fornecidas pelos idosos com o devido consentimento, as entrevistas foram registradas com o auxílio de um gravador de voz digital e transcritas imediatamente após sua realização. Este procedimento possibilitou o retorno ao material sempre que se fez necessário.

A apuração dos dados qualitativos foi realizada segundo a técnica da análise de conteúdo, de Bardin (BARDIN, 2009). Num primeiro momento, a análise 
das entrevistas foi realizada de forma independente pelos pesquisadores. Em seguida, os pesquisadores se reuniram com a finalidade de discutir os pontos de concordância e divergência em suas observações. Este procedimento teve como propósito reduzir a possibilidade de vieses oriundos da subjetividade e pela percepção seletiva que poderia ocorrer se um único indivíduo fosse responsável por todo o processo de análise. Os dados quantitativos coletados foram processados utilizando-se o software Microsoft Excel ${ }^{\circledR}$ 2007. Realizou-se análise estatística descritiva, com a distribuição da frequência das variáveis quantitativas.

Em conformidade com a Resolução n. 196/96 do Ministério da Saúde, para o desenvolvimento deste trabalho foi obtida a autorização da Secretaria Municipal de Saúde do Município de Governador Valadares, responsável pelas unidades de ESF selecionadas. Para sua realização, o trabalho obteve parecer favorável do Comitê de Ética em Pesquisa (CEP) da Universidade Vale do Rio Doce (UNIVALE), segundo o Parecer n. 028/09-10. Todos os idosos que participaram do estudo leram e assinaram o termo de consentimento livre e esclarecido (TCLE) em duas vias. Com o intuito de preservar a identidade dos entrevistados, os fragmentos das entrevistas apresentados neste trabalho foram identificados por "entrevistado" e o número correspondente à entrevista.

\section{Resultados}

Com base na leitura exploratória dos discursos, foram identificadas as seguintes categorias: 1) Prática e conhecimento; 2) Medidas higienodietéticas, que foi desagregada em três subcategorias: Alimentação; Exercícios físicos; Estresse e nervosismo; e 3) Tratamento medicamentoso.

\section{Categoria 1 - Prática e conhecimento}

Esta categoria aborda o conhecimento especulativo, racional do idoso sobre a terapêutica recomendada e sua ação de adesão ou não ao tratamento medicamentoso.

Observou-se existir diferenças entre as contribuições nos dois grupos de idosos quando se leva em consideração as medidas higienodietéticas e a terapia medicamentosa. Ou seja, o G1, em seu discurso, deu maior ênfase ao tratamento não-medicamentoso, enquanto o G2, na maioria das vezes, deu ênfase maior ao tratamento medicamentoso. Apesar das diferenças encontradas, constatou- 
se que os dois grupos possuem conhecimentos em relação à doença e ao tratamento. Contudo, tal conhecimento é expresso de forma distinta, como pode ser observado nos fragmentos apresentados. No G1, sempre que citavam uma medida de controle da HAS, diziam-se praticantes dela.

A gente num deve parar de tomar o remédio nunca. Tem que tomar o remédio direitinho, constantemente. Não pode parar. E sempre consultar com o médico, né? pra ver se aquele remédio precisa trocar ou se precisa deixar aquele mesmo. Continua com aquele mesmo. Que eu já troquei os meus. Já tomei Captopril, já tomei aquele [...], já tomei, agora tô tomando Propanolol. Mas tem muito tempo que eu não tomo. Tomo Propanolol com Hidroclorotiazida. Não [...] Tem que ver a alimentação, né? Alimentação mais sem gordura, não comer muita fritura, não comer comida muito temperada com sal, né? Comer verdura, essas coisas que ajudam mais, né? Comer menos coisa gorda porque tem gente que fica comendo comida gorda, temperada, taca sal na comida, e não deve fazer isso. Eu não faço. (Entrevistado 22 - Grupo G1).

Essa situação não foi observada no grupo G2, pois, apesar de os idosos demonstrarem possuir conhecimento em relação ao tratamento e às condutas adequadas para controle da pressão, durante o discurso deixavam transparecer que apesar de conhecerem o que deveria ser feito, não agiam em conformidade com tal conhecimento.

Tomar água de açúcar, para acalmar os nervos, tomar um chá pra não beber só remédio [...] a gente faz regime, também não come bolo, chocolate, deve comer frango [...] aí eu faço tudo certinho [...] só que ainda eu faço arte (Entrevistado 28 - Grupo G2)."

\section{Categoria 2 - Medidas Higienodietéticas}

Medidas higienodietéticas se referem a ações não-farmacológicas utilizadas na redução dos níveis pressóricos. Incluem a dieta com restrição de sal, redução do peso corporal, adoção de uma atividade física, moderação com bebidas alcoólicas, abolição do tabagismo e medidas antiestresse.

Apesar de existirem diferenças na ênfase com a qual foram citadas as medidas higienodietéticas nos discursos dos idosos, observa-se que o conteúdo das falas não apresenta grandes diferenciações. Por outro lado, os participantes concordam que os idosos com condição socioeconômica mais desfavorável possuem maiores dificuldades em fazer uma alimentação de qualidade, pois, segundo os próprios idosos, os alimentos saudáveis são mais caros.

[...] Ah! Alimentação tem que fazer, né? Sal eu como pouco. Tem que tomar o remédio direitinho, né? Se não tomar, piora, né? Eles dizem pra alimentar bem. Pobre não pode alimentar bem não. (Entrevistado 55 - Grupo G1) 
Em relação aos fatores sociodemográficos, observa-se na tabela 1 que o G2 possui maior proporção de idosos com idade em torno de 70 a 80 anos (43,5\%), classe socioeconômica mais baixa (64,2\%), D e E, e maior proporção de indivíduos de raça negra (37,1\%). Possivelmente, essas condições contribuíram para uma alta taxa de não-adesão ao tratamento entre os idosos hipertensos deste grupo.

Tabela 1. Fatores sociodemográficos associados ao nível de adesão ao tratamento em idosos de um distrito rural de Governador Valadares - MG

\begin{tabular}{|c|c|c|c|c|}
\hline \multirow[t]{2}{*}{ Fatores sociodemográficos } & \multicolumn{2}{|c|}{ Grupo G1 } & \multicolumn{2}{|c|}{ Grupo G2 } \\
\hline & Freq. & $\%$ & Freq. & $\%$ \\
\hline \multicolumn{5}{|l|}{ Idade (em anos) } \\
\hline 70 a 80 & 13 & 35,1 & 27 & 43,5 \\
\hline 80 e mais & 6 & 16,2 & 5 & 8,1 \\
\hline \multicolumn{5}{|l|}{ Sexo } \\
\hline Masculino & 11 & 29,7 & 26 & 41,9 \\
\hline Feminino & 26 & 70,3 & 36 & 58,1 \\
\hline
\end{tabular}

Situação de trabalho

Aposentado

19

51,4

32

66,1

Em exercício

7

18,9

30

8,1

Sem Vinculo

11

29,7

25,8

\section{Raça/Cor}

Negra

10

27,0

23

27,1

Outras

27

73,0

39

62,9

\section{Classificação da classe Socioeconômica}

\begin{tabular}{ccccc} 
B & 1 & 2,7 & 0 & 0,0 \\
$\mathrm{C}$ & 17 & 45,9 & 21 & 33,9 \\
$\mathrm{D}$ & 16 & 43,2 & 36 & 58,1 \\
$\mathrm{E}$ & 3 & 8,1 & 5 & 8,1 \\
\hline
\end{tabular}




\section{Subcategoria - Exercícios Físicos}

Nesta subcategoria, tanto o grupo G1 quanto o G2 se dizem praticantes de exercícios físicos. No entanto, observa-se que, em ambos os grupos, a realização destes exercícios é considerada desagradável.

O médico falou comigo assim, que eu tenho que tomar os remédios certos, né?! [...] Fazer caminhada, caminhada. Mandou eu fazer caminhada, né?! (Entrevistado 82 - G1).

\section{Subcategoria - Estresse e Nervosismo}

Este item aborda questóes relativas a sintomas que podem interferir nos níveis pressóricos do idoso, ligados às questôes psicológicas.

O fato de associar a HAS a sintomas psicológicos pode dificultar seu controle, pois os idosos deixam de tomar a medicação por acreditarem que esta conduta não é necessária. Segundo eles, tal situação se deve ao fato de a doença possuir período curto e passageiro, ocasionada por desentendimento com os filhos, esposa ou marido. Sendo assim, consideram que a melhor forma de tratamento é dormir bem e/ou conversar com parentes e amigos para "tirar o estresse".

O que me faz ter a pressão alta é a raiva, medo [...] tô passando raiva de mais [...] ai alguém me faz alguma coisa pra eu ficar muito nervoso [...] mas eu acho que a pressão me deixa mais nervoso, acho que sim, nada me faz raiva! (Entrevistado - Grupo 13)

Eu fico parado [...] às vezes deito um pouco pra ver se esquece as coisa ruim que acontece, saio pra conversar com a vizinha e desabafar as coisas que os filhos fazem e nos deixam com raiva [...] também tomo folha de carambola, vinagreira, limão com alho e outros 'remédio' do mato [...] Eu tenho que cuidar, olhar a pressão umas três vezes por semana. (Entrevistado 11 - Grupo G1).

\section{Categoria 3 - Terapia Medicamentosa}

Esta categoria está relacionada ao método de tratamento utilizado, bem como à percepção em relação aos medicamentos necessários no controle da HAS.

Outro fator que possivelmente pode interferir na forma como os pacientes fazem seu tratamento é a percepção da HAS como uma doença sintomática. Em alguns discursos do grupo G2, foi possível observar que o esquecimento da tomada da medicação estava associado a não apresentar sintomas que poderiam ser associados ao aumento da PA. Tal situação leva o paciente a desistir da terapia, 
já que o "sentir nada" é compreendido como a ausência de doença - sendo assim, é desnecessário o uso de medicamento.

Eu tomo mais esse Captopril pra controlar a pressão. Mas eu sou muito relaxado com esse negócio de tomar remédio. Porque eu não sinto nada, então eu venho aqui no Posto, por exemplo, e falam que minha pressão tá alta. Aí pego o remédio. Tomo um, dois comprimidos e largo aquilo pra lá. Pra mim eu num tô sentindo nada, né? Eu sei que a pressão não tá boa, mas eu não tenho aquele capricho de tomar o remédio. Eles dizem: você vai tomar todo dia, um de manhã, outro de tarde, à noite, por exemplo, um de manhã, outro à noite pra controlar a pressão. Eu tomo ali um dia, dois e largo aquilo pra lá e pronto. (Entrevistado 42 - Grupo G2).

Além deste fator, é possível identificar no grupo G2 a interferência negativa que a bebida alcoólica produz durante o tratamento medicamentoso da HAS. Os idosos desse grupo deixam de fazer uso da medicação para ingerir bebidas alcoólicas.

[...] Eu mesmo sou um dos que não cuida, né?! [..] Então às vezes eu só tomo o remédio e levo o remédio pra comadre [..] O remédio de pressão, ah, não cuido, porque não tomo remédio pra pressão e tomo a pinga, né [...] Aí não cuida, né? (Entrevistado 15 - Grupo G2).

Por outro lado, algumas percepções desta categoria se assemelham entre os dois grupos aqui analisados. Um ponto em comum é o fato de o estado fisiológico e biológico dos idosos parecer dificultar o tratamento e adesão aos medicamentos e às medidas gerais de controle da HAS. Alguns relataram a dificuldade em relação ao número de medicamentos e ao esquecimento no uso desses medicamentos, devido à complexidade de alguns regimes terapêuticos.

Tomo o remédio, cachaça não pode. Não faço mais nada [...]. Eu acho difícil de controlar porque tenho muito remédio que eu tomo. (Entrevistado 55 - Grupo G1).

Outro ponto a se destacar em ambos os grupos identificados neste estudo está relacionado às práticas culturais da região em relação ao tratamento da HAS. Estas práticas tendem a dificultar o tratamento da HAS, já que, para a maioria dos idosos, tão bom quanto os medicamentos prescritos pela equipe médica é o chá de origem caseira produzido com folhas ou frutos da região. Destes, temos vários exemplos como chás de carambola, amora, chuchu, erva-doce, para tudo, babosa, algodão, erva cidreira, vinagreira, limão, alho, “[...] suco de couve com três gotinhas de limão [...]", dentre outros.

Tomo chá de folha de abacate e também como muito chuchu e cebola [...], quando como cebola é bobeira eu toma o remédio, né? [...] (Entrevistado 61 - Grupo G1). 


\section{Discussão}

Pode-se considerar que um dos maiores desafios no atendimento aos hipertensos está relacionado ao seguimento da terapia prescrita pela equipe de saúde. Apesar de possuírem conhecimentos sobre a doença e seu tratamento, muitos pacientes não mantêm o controle da PA. A incongruência entre ter informação a respeito da doença e manter o controle da PA aponta para a diferença essencial entre conhecimento e prática, ou seja, apesar de conhecerem o que deve ser feito, os pacientes não agem em conformidade com tal saber. Em consonância, observouse entre os participantes do presente estudo que tanto os aderentes ao tratamento quanto os não-aderentes apresentaram conhecimento sobre a HAS. O que difere entre ambos é a prática deste conhecimento. Enquanto o conhecimento é racional, a adesão é um processo multifatorial influenciado por fatores emocionais, sociais, biológicos e culturais (VAN WIJK et al., 2005; ISRAELI et al., 2007).

Em relação ao conhecimento apresentado pelos pacientes, observa-se na literatura uma situação conflitante entre os autores. Enquanto alguns defendem que quanto maior o grau de conhecimento do paciente sobre sua doença, maior o comprometimento no autocuidado e adesão ao tratamento (JARDIM et al., 1996), outros afirmam que a adesão ao tratamento é uma questão mais complexa, pois dispor de acesso a informações sobre a gravidade, complicaçôes, recursos preventivos e terapêuticos da hipertensão não implica necessariamente maior adesão (AQUINO et al., 2001). Tendo como alicerce o segundo grupo de autores, observa-se que, entre os idosos do presente estudo, o conhecimento sobre as formas de tratamento não foi decisivo para sua adesão ao tratamento. O G2, apesar de possuir conhecimento sobre as formas de tratamento, não praticavam a adesão.

Estudosindicamacondiçãosocioeconômicacomoum preditordoconhecimento sobre a doença. Quanto mais baixos os níveis, menor o conhecimento da doença e mais difícil o acesso aos serviços de saúde e, consequentemente, menores as taxas de adesão ao tratamento anti-hipertensivo. Os sujeitos participantes da pesquisa acreditam que as baixas condiçôes socioeconômicas influenciariam no controle da PA na medida em que, segundo eles, os alimentos saudáveis e eficientes no controle da PA são mais caros. Ressalta-se que indivíduos com baixo poder aquisitivo (classes $\mathrm{C}, \mathrm{D}$ e E) apresentam maior risco de não-adesão, como já constatado em outros estudos (LESSA; FONSECA, 1997; MACHADO, 
2008). Observa-se na literatura que associações entre baixa classe econômica,

baixa escolaridade e cor negra sugerem que essas variáveis se apresentam como marcadores da não-adesão ao tratamento.

Quando comparada a adesão ao tratamento de pacientes com renda mensal menor que um salário mínimo aos que possuem renda mensal superior a três salários mínimos, observa-se uma diferença na percepção dos danos ocasionados pelo consumo de álcool ao tratamento. Ou seja, as pessoas com piores condições socioeconômicas acreditam que apenas com a diminuição do consumo de bebidas alcoólicas, a pressão arterial poderá ser reduzida. Além disso, o status econômico influencia na habilidade das pessoas em seguir as recomendações dos profissionais de saúde, não só no que se relaciona à compra de medicamentos, mas também no que se refere a fatores ligados aos aspectos educacionais, culturais, sociais e a mudança de hábitos de vida, como por exemplo, prática de atividade física e nãoingestão de bebida alcoólica (CAR et al., 1991; CLARK et al., 2000).

A prevenção e o tratamento da hipertensão por meio de intervenções nãomedicamentosas vêm conquistando diversos simpatizantes. Os profissionais de saúde indicam aos pacientes a utilização desta estratégia terapêutica com mais frequência, para assim desfrutarem de seus benefícios a médio e longo prazos. Dentre essas intervenções, está a prática de exercícios físicos. Alguns estudos sugerem que os tratamentos que incluem este tipo de intervenção alcançaram reduções na pressão arterial variando entre 5 e 21 mmHg (OSTERBERG; BLASCHKE, 2005). Apesar das vantagens em relação às intervenções que adotam os exercícios físicos, observou-se que os idosos do presente estudo demonstraram possuir restrições quanto a sua prática, mesmo após a orientação e autorização dos profissionais de saúde.

Quanto ao tratamento farmacológico, pode-se afirmar que, dentre os fatores que interferem na adesão, estão a assintomatologia da doença e a quantidade de medicações ingeridas diariamente. Em estudo realizado por Osterberg e Blaschke (2005), mais da metade dos pacientes (54,7\%) não utilizavam a medicação regularmente, propiciando a não-adesão ao tratamento. Estudos indicam que, quanto mais simples o esquema terapêutico, maior é a adesão ao tratamento (COELHO; NOBRE, 2006). Além disso, doses elevadas de medicamentos podem aumentar a incidência de reações adversas, sendo aversivo ao hipertenso continuar seguindo a terapia medicamentosa (COELHO; NOBRE, 2006). 
De modo geral, a despeito do fato de não gostarem do regime terapêutico em decorrência das muitas mudanças que o regime traz em suas vidas, os hipertensos consideram que devem fazer o tratamento. Assim, no início, procuram segui-lo à risca. É certo que os idosos tentam mudar seus hábitos de alimentação, habituarse a fazer exercícios, tomar as medicações regularmente, não fumar, entre outras modificaçōes. Mas, ao longo do tempo, muitas dessas tentativas se frustram e a maioria decide seguir o tratamento à sua maneira, considerando o que é possível fazer, o que consegue fazer ou o que quer fazer (REINERS; NOGUEIRA, 2009). Administrar o tratamento à sua maneira compreende ações como decisão de não tomar a medicação ou tomá-la apenas quando tiver sintomas, manipulação dos horários prescritos, escolha de atividades que mais lhe agradam, uso de práticas alternativas, decisão de parar com o tratamento, entre outras (CLARK et al., 2000). Alguns autores assinalam que a cronicidade da doença e a falta de sintomas são fatores importantes para o abandono do tratamento (LESSA; FONSECA, 1997; BUSNELLO et al. 2001; MASCARENHAS et al., 2006). Isto permite afirmar que, por não "sentir nada", ou seja, por não terem sintomas que associassem à HAS, os idosos deixam de adotar os cuidados adequados.

Outro ponto a se destacar é a influência dos estados emocionais do paciente na adesão ao tratamento. Em oposição a alguns trabalhos já destacados, Mallion \& Schmitt (2001) afirmam que a idade e a classe social têm pouca influência sobre a adesão do paciente. Características psicológicas desfavoráveis levam pacientes a perceber a HAS como uma consequência do estresse e ansiedade e, portanto, não seria necessário realizar um tratamento específico, apenas manter o controle emocional. O fato de associar a HAS a sintomas psicológicos como nervosismo, raiva, insônia e tristeza pode estar dificultando seu controle, pois os idosos deixam de tomar a medicação por acreditarem que esta conduta não é necessária para o controle da HAS.

O controle da HAS depende de medidas dietéticas e de estilo de vida e, quando necessário, do uso regular de medicamentos. No entanto, os idosos do presente estudo estão negligenciando tal cuidado. Por conseguinte, as atitudes demonstradas contribuem para que a HAS se torne importante causa direta ou indireta da morbidade e mortalidade por doenças do aparelho circulatório. Dessa forma, a análise e o monitoramento das condições de saúde da população, principalmente a idosa, são instrumentos essenciais para uma formulação 
equilibrada das políticas de saúde. Embora indicadores sintéticos de saúde sejam

úteis em determinados contextos, dispor de ferramentas que avaliem diferentes dimensões do processo saúde-doença propicia gerar formulações sobre um cenário mais completo sobre a HAS. Tal perspectiva, aplicada aos serviços públicos de saúde, pode auxiliar os profissionais na identificação do perfil e percepções da população sobre a HAS, auxiliando na promoção de ações em saúde com o objetivo de diminuir a incidência da HAS entre os jovens, os futuros idosos.

\section{Considerações finais}

Deve-se atentar para o fato de que os idosos hipertensos não aderentes ao tratamento tendem a considerar a HAS como uma doença aguda, sintomática e ligada a estados emocionais. Tal percepção equivocada torna necessária imediata reavaliação das orientações fornecidas aos hipertensos pela equipe de saúde, não bastando informar que a HAS é uma doença crônica e assintomática. Como observado, os pacientes possuem conhecimentos adequados acerca da HAS e do tratamento, mas são impulsionados a agirem em conformidade com suas opiniões e experiências socialmente compartilhadas.

Por fim, sendo o idoso mais propenso à HAS, deve-se dar especial atenção aos fatores que interferem no processo de sua adesão ao tratamento. Isto é, objetivando promover melhor qualidade de vida para este grupo populacional, os profissionais de saúde, inseridos em uma equipe multiprofissional, devem atentar para o nível de conhecimento destes sobre a HAS; a quantidade de medicações ingeridas; o uso de bebidas alcoólicas, o esquecimento; e as mudanças de estilo de vida, tais como o seguimento da dieta e prática de atividades físicas. ${ }^{2}$

\section{Agradecimentos}

Nosso sincero reconhecimento e agradecimento à Professora Dra ${ }^{\mathrm{a}}$. Claudia Medina Coeli, pela revisão e colaboração no manuscrito.

\section{Referências}

AQUINO, E.M.M.L.L. et al. Hypertension in a female nursing staff-pattern of occurrence, diagnosis, and treatment. Arq. Bras. Cardiol., v.76, n.3, p.203-08, 2001.

BARDIN, L. Análise de conteúdo. Lisboa: Ediçōes 70, 2009. 
BAUER, M.W.; AARTS, B. A construção do corpus: um princípio para a coleta de dados qualitativos. In: BAUER, M.; GASKELL, G. (Org.). Pesquisa qualitativa com texto, imagem e som. Petrópolis: Vozes; 2002. p. 39-63.

BUSNELLO, R.G. et al. Características associadas ao abandono do acompanhamento de pacientes hipertensos atendidos em um ambulatório de referência. Arq Bras Cardiol., v.76, n.5, p.349-51, 2001.

CAR, M.R.; PIERIN, A.M.G.; AQUINO, V.L.A. Estudo sobre a influência do processo educativo no controle da hipertensão arterial. Rev Esc Enf USP, v.25, n.3, p.255-69, 1991.

CARVALHO, J.A.M.; GARCIA, R.A. O envelhecimento da população brasileira: um enfoque demográfico. Cad. Saúde Pública. Rio de Janeiro, v.19, n.3, p.725-33, 2003.

CARVALHO, J.A.M.; RODRIGUEZ-WONG, L.L. A transição da estrutura etária da população brasileira na primeira metade do século XXI. Cad Saúde Pública. Rio de Janeiro, v.24, n.3, p.597-605, 2008

CHOBANIAN, A.V. et al. The Seventh Report of the Joint National Committee on Prevention, Detection, Evaluation, and Treatment of High Blood Pressure: the JNC 7 report. JAMA, n.239, p.2.560-72, 2003.

CLARK, M.J.; CURRAN, C.; NOJI, A. The effects of community health nurse monitoring on hypertension identification and control. Public Health Nursing, v.17, n.6, p.452-59, 2000. COELHO, E.B.; NOBRE, F. Recomendações práticas para se evitar o abandono do tratamento anti-hipertensivo. Rev Bras Hipertens, v.13, n.1, p.51-4, 2006.

FIRMO, J.O.A.; UCHOA, E.; LIMA-COSTA, M.F. The Bambui Health and Aging Study (BHAS): factors associated with the treatment of hypertension in older adults in the community. Cad. Saúde Pública. Rio de Janeiro, v.19, n.3, p.512-21, 2003.

INSTITUTO BRASILEIRO DE GEOGRAFIA E ESTATÍSTICA. Perfil dos idosos responsáveis pelos domicílios no Brasil 2000. Rio de Janeiro: IBGE, 2003.

ISRAELI, Z.H.; HERNÁNDEZ-HERNÁNDEZ, R.; VALASCO, M. The future of antihypertensive treatment. Am J Ther., v.14, n.2, p.121-34, 2007.

JARDIM, P.C.B.V.; SOUZA, A.L.L.; MONEGO, E.T. Atendimento multiprofissional ao paciente hipertenso. Medicina, v.29, p.29:232-8, 1996.

JOINT NATIONAL COMMITTEE ON PREVENTION, DETECTION, EVALUATION, AND TREATMENT OF HIGH BLOOD PRESSURE. The Sixth Report of the Joint National Committee on Prevention, Detection, Evaluation, and Treatment of High Blood Pressure. Arch. Intern. Med., n.157, p.2.413-46, 1997.

LESSA, I.; FONSECA, J. Raça, aderência ao tratamento e/ou consultas e controle da hipertensão arterial. Arq Bras Cardiol., v.68, n.6, p.443-9, 1997.

MACHADO, C.A. Adesão ao tratamento: tema cada vez mais atual. Rev Bras Hipertensão, v.15, n.4, p.220-21, 2008. 
MALLION, J.M., SCHMITT, D. Patient compliance in the treatment of arterial hypertension. Blood Press, v.11, n.4, p.253-4, 2002.

MASCARENHAS, C.H.M.; OLIVEIRA, M.M.L.; SOUZA, M.S. Adesão ao tratamento no grupo de hipertensos do Bairro Joaquim Romão - Jequié/BA. Rev Saúde Com., v.2, n.1, p.10-38, 2006.

MINAYO, M.C.S. Cultura: tradição e contradição, uma contribuição da antropologia para se pensar educação e saúde. In: MELO, J.A.C. (Org.). Educação: razão e paixão. Rio de Janeiro: Panorama, 1993.

MORISKY, D.E.; GREEN, L.W.; LEVINE, D.M. Concurrent and predictive validity of a self-reported measure of medication adherence. Med Care, v.24, p.67-74, 1986.

OSTERBERG, L.; BLASCHKE, T. Drug therapy: adherence to medication. NEJM, n.353, p.487-97, 2005.

PERES, D.S.; MAGNA, J.M.; VIANA, L.A. Arterial hypertension patients: attitudes, beliefs, perceptions, thoughts and practices. Rev. Saúde Pública. São Paulo, v.37, n.5, p.635-42, 2003.

REINERS, A.A.O.; NOGUEIRA, M.S. Conscientização do usuário hipertenso para a adesão ao tratamento. Rev Latino-am Enfermagem, v.17, n.1, p.59-65, 2009.

SANTOS, Z.M.S.A. et al. Adesão do cliente hipertenso ao tratamento: análise com abordagem interdisciplinar. Texto Contexto - Enferm., v.14, n.3, p.332-40, 2005.

SERAPIONI, M. Métodos qualitativos e quantitativos na pesquisa social em saúde: algumas estratégias para interação. Ciênc. Saúde Col., v.5, n.1, p.187-92, 2000.

TERRA, D.F. et al. Redução da pressão arterial e do duplo produto de repouso após treinamento resistido em idosas hipertensas. Arq. Bras. Cardiol., v.91, n.5, p.299-305, 2008. VAN WIJK, B.L. et al. Rate and determinants of 10-year persistence with antihypertensive drugs. J Hypertens., v.23, n.11, p.2.101-07, 2005.

WORLD HEALTH ORGANIZATION. The world health report 2008 - primary health care (now more than ever). Geneva: WHO, 2008.

ZAITUNE, M.P.A. et al. Hipertensão arterial em idosos: prevalência, fatores associados e práticas de controle no Município de Campinas, São Paulo, Brasil. Cad. Saúde Pública. Rio de Janeiro, v.22, n.2, p.285-94, 2006.

\section{Notas}

${ }^{1}$ Este artigo é um recorte da pesquisa "Portadores de Hipertensão Arterial Sistêmica: representações dos pacientes a respeito da doença", fomentada pela Fundação de Amparo à Pesquisa de Minas Gerais (FAPEMIG).

${ }^{2}$ L.O. Leão e Silva, M.M. Soares e M.C. de Oliveira participaram da concepção, delineamento, análise e interpretação dos dados; redação do artigo. S.M. Rodrigues, C.J. Machado e C.A. Dias participaram da análise e interpretação dos dados; redação do artigo e sua revisão crítica; aprovação da versão a ser publicada. 


\section{Abstract}

\section{"I feel nothing": perceptions of elderly patients on the treatment of hypertension}

This paper aims to identify the perceptions of elderly hypertensive patients about therapy, related or not to adherence to medication. The qualitative sample consisted of 16 elderly of both sexes, aged 60 and older living in two rural districts of the municipality of Governador Valadares, Brazil, who used some medication to control blood pressure and had independent functional status and cognitive function preserved. Data was collected through open interview at depth, with the aid of a digital voice recorder, conducted between June and September 2010. The assessment of information obtained was performed according to the technique of content analysis of Bardin, which found these categories: Practice and knowledge, Hygienic and dietetic Measures (Food, Exercise, Stress and nervousness) and drug treatment. The results showed that older people have adequate knowledge about hypertension and treatment, but are driven, during therapy, to act according to their socially shared opinions and experiences. Moreover, elderly hypertensive non-adherent tend to consider hypertension as an acute, symptomatic and linked to emotional states. We conclude that, because of this misperception, it is necessary to reevaluate the guidance given to hypertensive patients by the health staff, and viable alternatives to promote health must be created, to change attitudes and practices regarding the disease.

> Key words: hypertension; therapeutics; elderly; adherence to medication. 